\title{
Mobile Application for Horse Training Program System
}

\author{
Amirul Asyraf Rosman', Hadafi Fitri Mohd Latip ${ }^{1,2 *}$ \\ ${ }^{1}$ School of Biomedical Engineering and Health Sciences, Faculty of Engineering, Universiti Teknologi Malaysia, \\ 81310 UTM Johor Bahru, Johor \\ ${ }^{2}$ Sport Innovation \& Technology Centre, Institute of Human Centered Engineering, Universiti Teknologi Malaysia, \\ 81310 UTM Johor Bahru, Johor \\ *Corresponding Author hadafifitri@utm.my
}

Received 26 October 2021; Accepted 27 January 2022; Available online 31 January 2022

https://doi.org/10.11113/humentech/v1n1.9

\begin{abstract}
:
Different equestrian sports may necessitate distinct training regimens or demands. Inadequate knowledge of appropriate and acceptable horse exercise regimes and training programs may result in horse injury and nonprogressive horse performance. It is critical to adopt exercise or training programs that are based on a correct training concept, as this can assist horse riders in having a proper planned training management and therefore developing their horse's performance. Mobile phone applications are so entwined in modern culture and are continuously accessible to users, they could be beneficial as mobile coaching systems targeted at raising physical activity levels. The goal of this research is to employ mobile application software to guide horse riders in conditioning their horses to optimal performance and to emphasize the need of using appropriate and accurate equipment. The theory of development of the app will be using ADDIE model. The mobile application was developed using the online software development tool; Kodular Creator.
\end{abstract}

Keywords: Equestrian; Mobile application; Training programs; Kodular Creator

\section{Introduction}

Horse training is an essential component of developing good horse physical condition. Horses must be in peak physical fitness and have the correct psychological state to achieve optimum individual performance within any equestrian discipline [1]. According to Federation Equestrian International (FEI), to optimize horse performance, proper fitness and training regime is key to building up cardiovascular and muscular strength. To constantly improve upon horse performance, it is essential to turn into a habit. This can only be possible with a consistent training regime and a commitment to a training plan. Regular training helps to fully immerse into a learning process and gives an impulse to further improvements towards achieving a goal.

Different equestrian sports may require different training programs or demands. In order to design a training program, it is important to be realistic about the horse's age, breed and fitness/injury rehab requirements, the type and level of sport demand, and the rider's own fitness and time commitment [2]. People are aware of the health benefits of physical activity, however the guidelines in practicing physical activity did not meet a large proportion of the population. Accordingly, simple and widely accessible approaches are required to increase physical activity rates [3]. This study contains the idea of providing horse exercise suggestions, and horse training program options.

There are two important condition that should be taken into consideration when training a horse which is fitness of the horse cardiovascular respiratory system and fitness of the musculoskeletal system [4]. A well-trained or a fit horse 
are able to complete a required amount of physical activity without having any issue of fatigueless, stress, or injuries. The horse must be kept safe and not undertrained or over-trained in the best way [5]. Fitness conditioning of horse as an athlete may involve few types which are the aerobic conditioning, anaerobic conditioning, and strength training [5]. The purpose of this study is to implement the use of mobile application software in guiding horse rider to condition their horse and applying suitable, and correct training regimes based on their sport demand. This study is conducted into application development study.

\section{Materials and Methods}

\subsection{Mobile application design}

The interface concept of this mobile application is graphical. The Home Page displays the list of function and features in the mobile application. On top of the page, various option tabs of categories are available to be chosen. The tab options are 'Home page', 'Exercise list page' and 'Specific exercise page'.

\subsection{Main Menu page}

The Main Menu page interface (Figure 1) displayed three choices of general category of horse training with an Enter button choice for user to choose which category they want to view. In this main menu page, there were three main categories of training which were 'Flatwork', 'Jumping' and 'Groundwork training'.

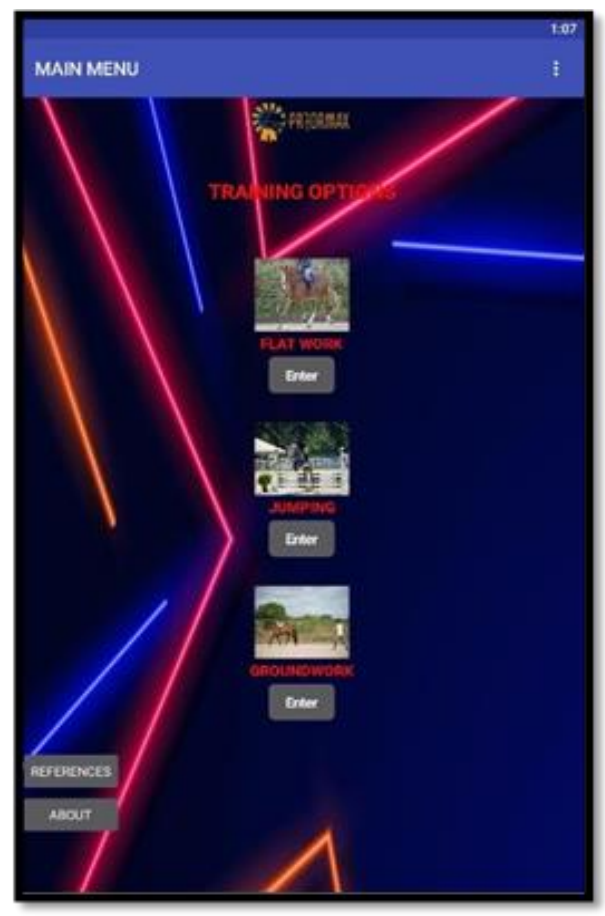

Figure 1. Main Menu page

\subsection{Exercise list page}

The interface layout (Figure 2) used to develop the list of exercises page interface are the Horizontal Arrangement Layout for the mobile app logo and home button at the top of the page. Next layout used are the Table Arrangement, whereby list of exercises image to indicate the exercise were arranged in vertical arrangement. Each exercises name was labelled under their respective image. Each exercise was also categorized based on their level of difficulties and there is arrow button besides each of the exercise to be directed to the chosen exercise page. 


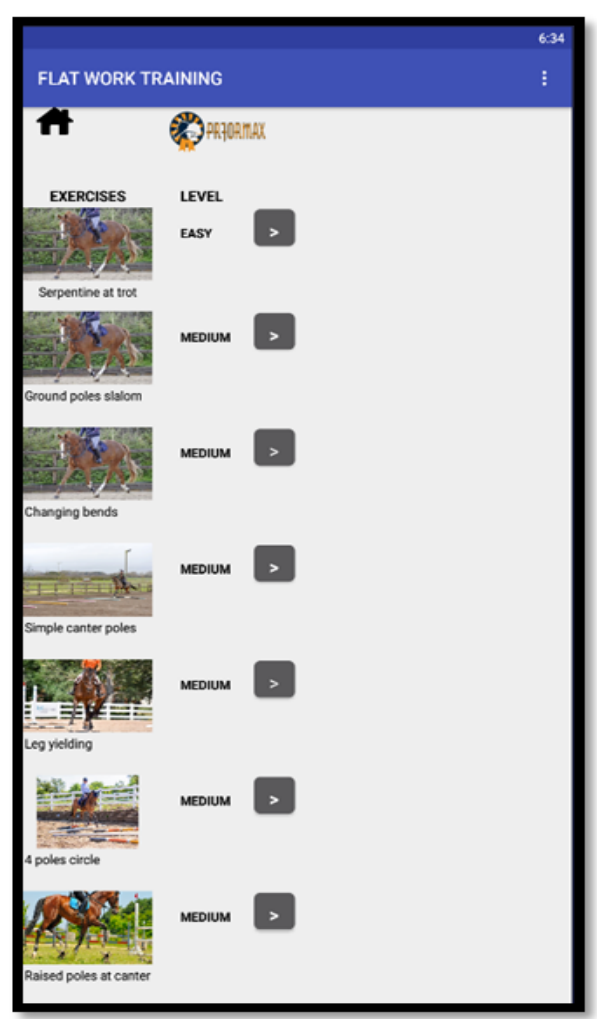

Figure 2. List of 'Exercise page' interface

\subsection{Specific exercise page}

As any of the exercise from the list are chosen, the page then redirected to the specific exercise page. At the top of the page there are name of the exercise. The layout used for the specific exercise page interface (Figure 3) were Scrollable Vertical Arrangement.

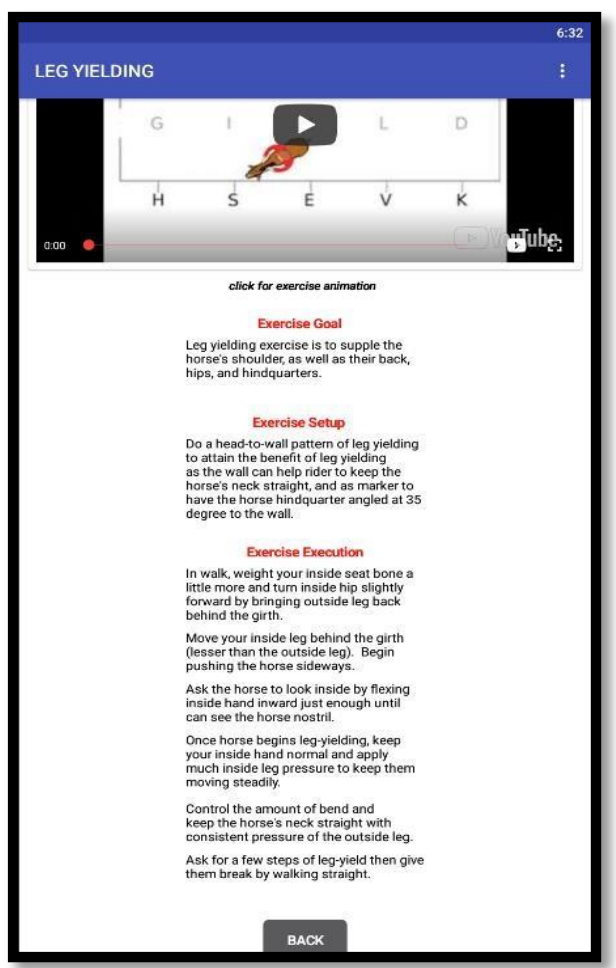

Figure 3. 'Specific exercise page' interface (c) 2022 Penerbit UTM Press. All rights reserved 
Image widgets are also inserted to display a picture regarding the overview layout of the exercise. Underneath the Image widget, Textbox widgets were placed to insert description of the Exercise Goal, Exercise Setup, and Exercise Execution. Content of the exercises are ranging from selection of training for different levels which are easy, medium, and hard, which includes sections on specific disciplines: dressage exercise, jumping exercise, and groundwork or lunging exercise.

\section{Results}

\subsection{Mobile application user experience}

When user opens this mobile app, a landing page will appear and loading for 5 seconds. Then, it will display the Main Menu Page (Figure 4) displayed the Training Options which were Flatwork, Jumping, and Groundwork, and there were two additional buttons at the bottom of the page which were the References button, and the About button.

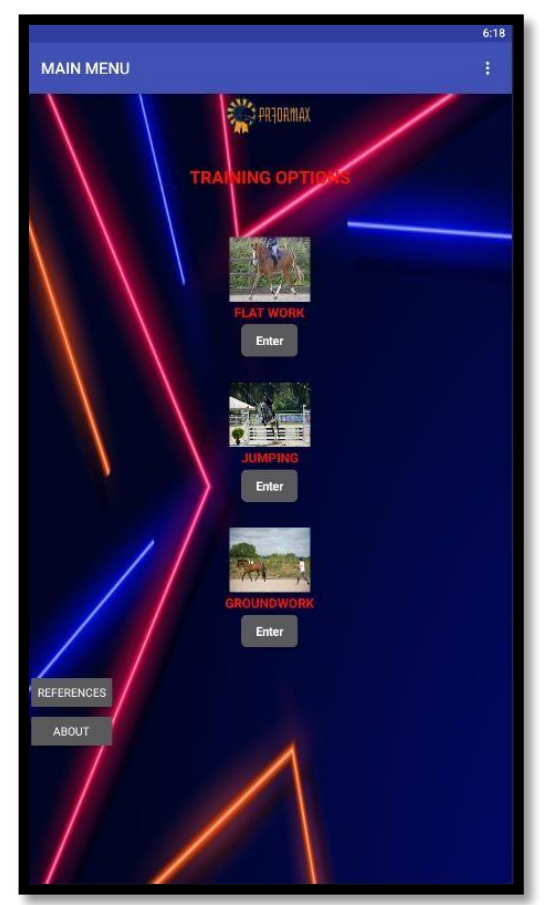

Figure 4. Main Menu page interface

Once user choses by clicking Enter in any Training Options, then it will go to next page which shows list of exercises to be performed as shown in Figure 5. In the list of exercises, once the user clicked on the arrow button beside the preferred exercise, it will then direct to a page where there will be display of the exercise layout image or video animation, the description of the exercise goal, the setup of the exercise, and the execution of the exercise. To choose another exercise, user can click on the back button at the bottom of the page as it will return to the list of exercises page.

\subsection{Installation interest result}

The question states "Will you install the mobile app if it is published? (Yes / No / Maybe)". This question was introduced to identify the interest among the respondents whether to install the Mobile Application for Horse Training or not. The result obtained from this question can be used determine the usability of the mobile app through the interest of the potential users of this mobile app which includes Bachelor of Science (Equine Management) students in Universiti Teknologi Malaysia, equine industry players in Malaysia, and public users in Malaysia.

Positive results had been achieved as most of the respondents answered "Yes" as they are willing to install the mobile app if it is published. Percentage of $82.5 \%$ or total of 33 people among the respondents are mixed from Bachelor of Science (Equine Management) students, equine industry player, and public users who are willing to install the mobile app if it is published. $10 \%$ of the respondents which were three from equine industry and one public user states that they 
"Maybe" install the mobile app, meanwhile the remaining 7.5\% or three public users answered "No" to install the mobile app.

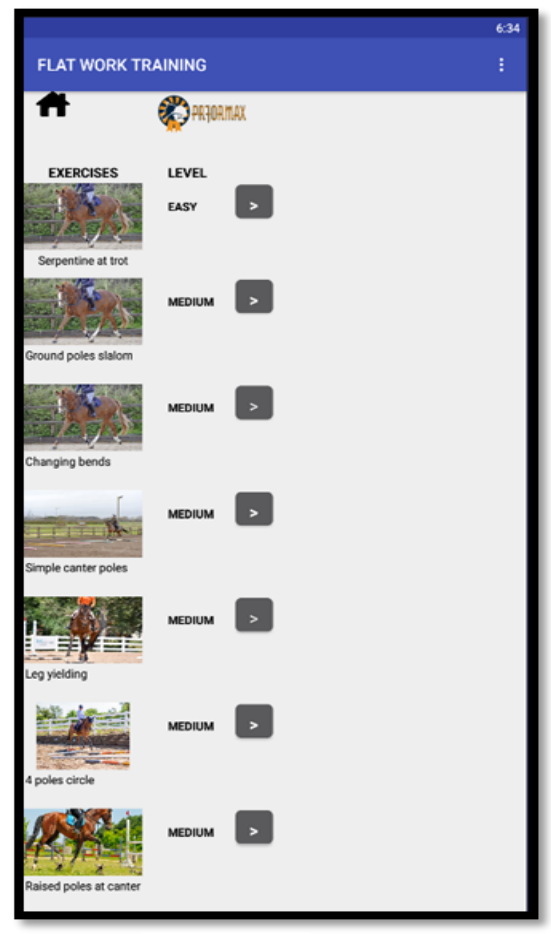

(a)

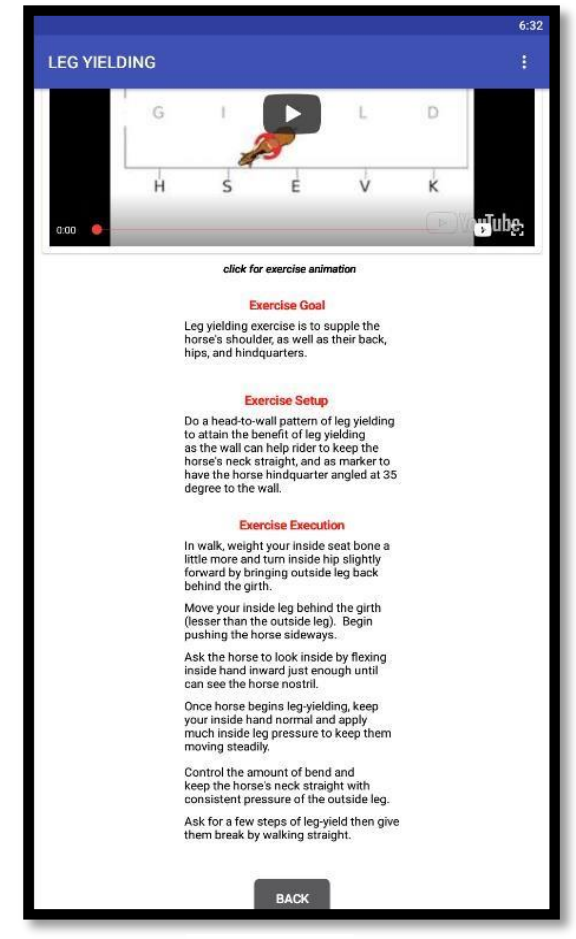

(b)

Figure 5. List of (a) Exercise page and (b) Specific exercise page

\section{Discussion}

Initial system testing is essential of the developed system because it can facilitate in distinguishing or discovering problems, bugs, logical errors, compilation errors or run-time errors. This testing helps to ensure the developed system are functioning and meet the necessities that had been set throughout the designing procedure. To proceed with the system testing procedure, user testing by using AI companion is applied to the Mobile Application for Horse Training.

Before proceeding to the system testing, AI companion for Kodular Creator is first installed in an Android device called the Kodular Companion. The Kodular Companion functioned to connect the designed mobile app to a device to be tested before it is published fully. Once companion had been completely installed, a QR code from the Kodular Creator are then generated to be scanned by the Android device through the Kodular Companion app. As the QR code been scanned, the Mobile Application for Horse Training Program will show up on the Android device.

\section{Conclusion}

People are ready and willing to use the mobile application as an alternative way to an accessible horse exercise options and horse training programs in easier way. The adopted exercises and training programs will effectively train horses by assisting horse riders for proper planned training management.

\section{References}

[1] S. D. McBride and D. S. Mills, Psychological factors affecting equine performance, BMC Veterinary Research, 2012, 8(180). https://doi.org/10.1186/1746-6148-8-180

[2] B. Henderson, Horse Canada, 2017, https://horsecanada.com/magazine/training/conditioning-strategies-all-horses/ [Accessed 22 December 2020]. 
[3] D. J. Peart, C. Balsalobre-Fernández and M. P. Shaw, The use of mobile applications to collect data in sport, health and exercise science: A narrative review, Journal of Strength and Conditioning Research, 2019, 33(4):1167-1177. https://doi.org/10.1519/jsc.0000000000002344

[4] C. Castejon-Riber, C. Riber, M. D. Rubio, E. Agüera and A. Munoz, Objectives, principles, and methods of strength training for horses, Journal of Equine Veterinary Science, 2017, 56:93-103. https://doi.org/10.1016/j.jevs.2017.04.011

[5] R. G. Lockie, M. D. Jeffriess, T. S. McGann, S. J. Callaghan and A. B. Schultz, Planned and reactive agility performance in semiprofessional and amateur basketball players. International Journal of Sports Physiology and Performance, 2014, 9(5):766-771. https://doi.org/10.1123/ijspp.2013-0324 